\title{
Separation of Xylose Oligomers from Autohydrolyzed Miscanthus $x$ giganteus using Centrifugal Partition Chromatography
}

\author{
Ming-Hsu Chen ${ }^{1,+}$, Kalavathy Rajan ${ }^{2,+}$, Danielle Julie Carrier ${ }^{3}$ and Vijay Singh ${ }^{1}$
}

1. Department of Agricultural and Biological Engineering, University of Illinois at UrbanaChampaign. 1304 W. Pennsylvania Avenue, Urbana, IL 61801, USA

2. Department of Food Science, University of Arkansas, 1180 W Cassatt St., Fayetteville, AR 72704, USA

3. Department of Biological and Agricultural Engineering, University of Arkansas, 203 Engineering Hall, Fayetteville, AR 72701, USA

+ Co-first author

*Corresponding author. Tel: 217-333-9510, Fax: 217-244-0323

E-mail address: vsingh@illinois.edu (Dr. Vijay Singh) 


\begin{abstract}
Autohydrolysis of cellulosic materials for saccharification generates xyloseoligosaccharides (XOS), due to the partial hydrolysis of xylan. Developing an efficient method for the separation and recovery of XOS from the prehydrolyzates would provide an excellent opportunity for the better utilization of the cellulosic material and for value-added co-product production. In this study, we investigated the use of centrifugal partition chromatography (CPC) for the fractionation of XOS from Miscanthus x giganteus (MxG). During autohydrolysis of miscanthus biomass at $180^{\circ} \mathrm{C}$ for $20 \mathrm{~min}, 63 \%$ of xylan was converted into XOS and xylose. The ensuing XOS concentrate contained up to $30 \%$ of XOS, which were distributed as $15.9 \%$ xylobiose (DP2), $5.9 \%$ xylotriose, (DP3), $5.6 \%$ xylotetraose (DP4), $0.8 \%$ xylopentaose (DP5) and $0.6 \%$ xylohexaose (DP6). The XOS concentrate was further fractionated by CPC with a solvent system composed of 4:1:4 (v/v/v) butanol: methanol: water. Using CPC techniques, 230 $\mathrm{mg}(80 \%)$ of DP2 to DP6 oligomers were fractionated from $1 \mathrm{~g}$ of XOS concentrate. The recoveries of individual XOS were 90.2 \% DP2, 64.5\% DP3, 71.2 \% DP4, $61.9 \%$ DP5 and 68.9 \% DP6. The purities of DP2 to DP6 fractions were 61.9\%, 63.2\%, 44.5\%, $31.5 \%$ and $51.3 \%$, respectively. Presence of DP2 and DP3 in the CPC purified fractions was further validated by mass spectrometry analysis. The study provided information on fast recovery of individual XOS from crude biomass prehydrolyzate.
\end{abstract}

\footnotetext{
Key words: Miscanthus x giganteus, Autohydrolysis, XOS, Xylose oligomers, Centrifugal partition chromatography
} 


\section{Introduction}

Development of cellulosic biomass for biofuels is making progress in the U.S.; according to a recent report, several commercial plants are expecting to start operation in 2014-15 (Brown and Brown, 2013). Using cellulosic biomass in fuel conversion has many benefits to the environment, energy security and rural economy (Kim and Kim, 2014). Among all bioenergy crops, Miscanthus x giganteus (MxG), a perennial rhizomatous grass, is a leading candidate for fuel production. MxG produces $60 \%$ more biomass than well-fertilized maize and the yield can reach $30 \mathrm{MT} / \mathrm{ha} / \mathrm{year}$ (Somerville et al., 2010). Over 50\% of the MxG cell wall consists of structural carbohydrates (cellulose and hemicellulose), which are available for biofuels and biochemicals production (Brosse et al., 2012).

The autohydrolysis involves treating biomass with hot water or steam (140 to $\left.200^{\circ} \mathrm{C}\right)$ to open the structure of plant cell wall. At high temperatures, water acts as a weak acid and releases acetyl groups from hemicellulose, which further acidifies the reaction mixture. These hydronium ions catalyze the cleavage of glycosidic bonds within the xylan, resulting in the liberation of xylose and other sugar oligomers into the liquid phase. The sugar oligomer enriched prehydrolyzate is generally washed off and the pretreated solid residue, which is enriched in cellulose, can be further processed to ethanol (Vázquez et al., 2000).

Xylose oligomers (XOS) are indigestible carbohydrates that find application in prebiotics, since they are selectively utilized by human gut microbiota like Bifidobacterium and Lactobacillus spp., thereby resulting in health benefits to the host (Carvalho et al., 2013). XOS have the potential of becoming value added co-products of the cellulosic ethanol industry. However, direct separation of XOS from the prehydrolyzate is a complex problem (Moure et al., 2006), because in addition to XOS, there may be other monomeric and oligomeric sugars, sugar 
degradation products, acid solubilized lignin and inorganic compounds (Gullón et al., 2010).

Multiple steps of separation including solvent extraction, vacuum evaporation, surface-

adsorption, membrane separation, size exclusion chromatography and ion exchange

chromatography have been reportedly used in sequential purification of XOS (Vázquez et al., 2000; Moure et al., 2006). XOS were purified from rice husk hydrolyzate using membrane concentration, endo-xylanase digestion, and anion exchange resins (Gullón et al., 2010). Activated carbon adsorption and ethanol elution was also used to recover XOS from MxG hydrolyzates (Chen et al., 2014). However, both Gullón et al. (2010) and Chen et al. (2014) recovered XOS mixtures, rather than purified oligomers that have a single degree of polymerization (DP).

Centrifugal partition chromatography (CPC) is a separation method developed from countercurrent chromatography. This liquid-liquid extraction technology uses two immiscible solvents as the stationary and mobile phase during chromatographic purification. Separation of solutes is achieved by virtue of their difference in partition coefficients. CPC has been widely used for single step preparative separations of alkaloids and flavonoids from crude plant extracts, for pharmaceutical applications (Attoumbre et al., 2012; Jeon et al, 2012). Advantages of CPC include the ability to separate compounds that span a wide molecular weight range, such as XOS (Berthod et al., 1988). Birchwood xylan-derived XOS were previously fractionated by CPC into its individual oligomers with a tetrahydrofuran-based solvent system (Lau et al., 2011). The CPC method was further improved with a butanol-based solvent system (Lau et al., 2013). However, these CPC protocols were never tested with crude hydrolyzates that stemmed from biomass autohydrolysis reactions. 
The objective of this study was to purify XOS from crude MxG hydrolyzates, which were produced through autohydrolysis, into their individual oligomer components. It is important to note that the starting material in this study consisted of a crude complex hydrolyzate, as opposed to a semi purified xylan material (Lau et al., 2011; Lau et al., 2013; Bunnell et al., 2015). Separation and fractionation of MxG XOS could possibly provide an opportunity to produce valuable co-products from bio-refineries, for pharmaceutical and nutraceutical applications.

\section{Material and methods}

\subsection{Raw material and chemicals}

Miscanthus $x$ giganteus (MxG) was grown at the Energy Farm, University of Illinois, Urbana, IL $\left(40.06^{\circ} \mathrm{N}, 88.19^{\circ} \mathrm{W}\right)$. The $\mathrm{MxG}$ was harvested in 2011 and was dried at $49^{\circ} \mathrm{C}$ in a convective oven, cut and ground in a hammer mill (model MHM4, Glen Mills, Clifton, NJ) to pass through a $250 \mu \mathrm{m}$ screen. The dried and ground $\mathrm{MxG}$ was stored at $4{ }^{\circ} \mathrm{C}$. The moisture content was 8.2\%, as determined following the NREL-LAP-001 protocol (Sluiter et al., 2008a). Xylose (DP1), arabinose, glucose and galactose were purchased from Sigma-Aldrich (St. Louis, MO). Xylobiose (DP2), xylotriose (DP3), xylotetraose (DP4), xylopentaose (DP5), and xylohexaose (DP6) were purchased from Megazyme International (Wicklow, Ireland) and their purity was reported to be greater than 95\%. High performance liquid chromatography (HPLC) grade butanol and methanol were obtained from Macron Fine Chemicals (Center Valley, PA) and EMD Chemicals (Gibbstown, NJ), respectively. Water was prepared with a Direct-Q system (Millipore, Billerica, MA). Formic acid (95\%) was procured from Amresco LLC (Solon, OH).

\subsection{Autohydrolysis and sample preparation}


The autohydrolysis was performed in a tubular reactor inserted in a fluidized bed sand bath for fast heating (Khullar et al., 2013). The 316 stainless steel tubing (SS-T12-S-065-20, Swagelok, Chicago Fluid System Technologies, Chicago, IL) reactors had an outside diameter of $19.1 \mathrm{~mm}$, a wall thickness of $1.7 \mathrm{~mm}$, and a length of $104.8 \mathrm{~mm}$. Reactors were capped with 19.1 mm, 316 stainless steel Swagelok caps (SS-1210-C, Swagelok) on both ends. The procedure was essentially as described by Chen et al. (2014), where the loaded reactors were immersed in a fluidized sand bath (IFB-51 Industrial Fluidized Bath, Techne Inc., Burlington, NJ) preheated to $180^{\circ} \mathrm{C}$ and cooled in flowing room temperature tap water. Autohydrolysis was performed at water to solid ratio of $9: 1$, at $180^{\circ} \mathrm{C}$ for $20 \mathrm{~min}$. In each tubular reactor, $5 \mathrm{~g}$ of $\mathrm{MxG}$ was mixed with $45 \mathrm{~g}$ of water. The pretreated solids and liquids were separated by centrifugation $(2600 \times g$, $5 \mathrm{~min}$ ) in an Eppendorf 5804R centrifuge. This procedure was repeated in six reactors for four batches and corresponding hydrolyzates were combined. The resulting volume, determined to be $420 \mathrm{~mL}$, was filtered through a $0.45 \mu \mathrm{m}$ filter membrane (Millipore, Bedford, MA) and dried in a $49^{\circ} \mathrm{C}$ oven, removing approximately $415 \mathrm{~mL}$ of water. The resulting crude dried mixture was subsequently referred to as "XOS concentrate" throughout the work.

\subsection{Sugar and oligomer characterization}

Xylose, glucose, arabinose, galactose and XOS (from DP2 to DP6) content in the XOS concentrate was determined using a Waters Alliance HPLC system (Model 2695, Waters Corporation, Milford, MA) equipped with SP0810 (Shodex, Kawasaki, Japan) or Bio-Rad Aminex-HPX 42A (Bio-Rad, Hercules, CA) columns for monomer and oligomer quantification, respectively. HPLC was equipped with SP-G pre-column for monomer and Micro-Guard Deashing pre-column for oligomer determinations. Purified and $0.22 \mu \mathrm{m}$ filtered water was used as eluent. Column temperature was $85^{\circ} \mathrm{C}$. Eluent flow rate was $0.2 \mathrm{~mL} / \mathrm{min}$, and monomers or 
oligomers were detected with a refractive index detector (Model 2414, Waters Corporation, Milford, MA), which was maintained at $50^{\circ} \mathrm{C}$. The concentrations of xylose, glucose, arabinose, galactose and XOS (DP2 to DP6) were determined using in-house calibration curves generated from commercial standards.

The total dissolved solids and sugar content of the concentrate were determined following NREL LAP-001 (Sluiter et al., 2008a) and NREL-LAP- 014 (Sluiter et al., 2008b) protocols, respectively. Accordingly, the total yield of xylose oligomers in the XOS concentrate was determined as follows:

$$
\text { Yield }(\%)=\left(W_{h}-W_{i}\right) \times 0.88 \times 100
$$

Where $W_{h}$ was the mass fraction of xylose in the concentrate after acid hydrolysis, $W_{\mathrm{i}}$ was the initial mass fraction of xylose in the concentrate and 0.88 is the anhydro correction factor for xylose (Sluiter et al., 2008c). The total phenolic content of the concentrate was determined using the Folin \& Ciocalteau's (F-C) method, based on a previously published protocol (Ainsworth and Gillespie, 2007).

\subsection{Centrifugal partition chromatography}

A bench scale SCPC-250 system from Armen Instruments (Dallas, TX) equipped with CherryOne Beta (C1) countercurrent chromatography control system (Chicago, IL) was used for fractionating and purifying the XOS concentrate. Samples were processed by ultrapure nitrogen at 43 psig and with an evaporative light scattering detector (ELSD) (SofTA Corp, Westminster, CO). Butanol: methanol: water solvent system in 4:1:4 (v/v/v) was used for the CPC separation (Lau et al., 2013). To prepare $2 \mathrm{~L}$ of the biphasic system, $889 \mathrm{~mL}$ of butanol was mixed with 222 $\mathrm{mL}$ of methanol and $889 \mathrm{~mL}$ of water in a separation funnel and agitated thoroughly. The upper butanol rich organic phase and the lower aqueous phase were collected separately after $4 \mathrm{~h}$ and 
used as the mobile and stationary phases, respectively, when the CPC was operated in the ascending mode. One g of dried XOS concentrate was dissolved in $20 \mathrm{~mL}$ of butanol-rich phase and $8 \mathrm{~mL}$ of the aqueous-rich phase. The mixture was then vortexed and filtered through a $1 \mu \mathrm{m}$ PTFE syringe filter (Thermo Scientific National, Rockwood, TN) and then injected into the CPC system.

The stationary and mobile phases were loaded in the ascending mode at 500 RPM. The equilibrium was reached at retention volumes of $114 \mathrm{~mL}$ and $136 \mathrm{~mL}$, respectively. The CPC run time was $344 \mathrm{~min}$, at $2300 \mathrm{RPM}$ and at a flow rate of $8.14 \mathrm{~mL} / \mathrm{min}$. The UV detector in the controller was equipped with a preparatory flow cell that was set at $254 \mathrm{~nm}$ for real-time monitoring. Fraction collection was started after $60 \mathrm{~min}$ and fractions of approximately $8.1 \mathrm{~mL}$ each, were collected every minute. To accomplish this task, two fraction collectors were connected in series: Foxy R1 (Teledyne Isco, Lincoln, NE) and Waters Fraction Collector III (Waters Corporation, Milford, MA). After 240 min of run time, the CPC was operated in the descending mode allowing for un-separated compounds to exit the column and be collected. All 284 CPC fractions were dried in a Savant SpeedVac Concentrator SPD 1010 (Thermo Scientific, Ashville, NC), at 7 Torr for $6 \mathrm{~h}$ and reconstituted in $0.5 \mathrm{~mL}$ of water. The reconstituted fractions were filtered through a $0.22 \mu \mathrm{m}$ nylon syringe filter (Thermo Scientific National, Rockwood, TN) and analyzed using HPLC to determine the concentration of xylose monomer and oligomers (DP2 to DP6).

The CPC fractionation of MxG XOS was repeated twice. Fractions were consolidated based on the concentration of each xylose oligomer. The purity of consolidated samples was calculated as:

$$
\% \text { Purity }=\frac{\text { Weight of } x y \text { lose oligomer }}{\text { Total weight of each fraction }} \times 100
$$




\subsection{ESI-MS verification}

Samples were dried in SpeedVac and mixed with $0.1 \%$ formic acid solution in methanol, at 20:1 ratio and directly injected into the mass spectrometer with a syringe pump. Gas phase ions created using electrospray ionization (ESI) were analyzed with a quadrupole/time -of flight (Q-TOF) mass analyzer (Bruker ultrOTOF-Q, Bruker Daltonics Inc., Billerica, MA). The solvent (methanol) flow rate was $3 \mu \mathrm{L} / \mathrm{min}$ and $\mathrm{N}_{2}$ gas at 1 bar was used for nebulization. The temperature of the source was $180^{\circ} \mathrm{C}$. Other instrument parameters were adjusted to optimize signal in the $m / z$ range of $100-800$ and the mass spectra were obtained in positive ion mode.

\section{Results}

\subsection{Production of XOS concentrate}

Miscanthus $x$ giganteus $(\mathrm{MxG})$ biomass composition, previously determined by Chen et al. (2014), was $35.9 \%$ glucan, $19.5 \%$ xylan, $18.5 \%$ acid insoluble lignin, $11.3 \%$ total extractives, $1.8 \%$ ash and $1.1 \%$ acid soluble lignin. Autohydrolysis of $\mathrm{MxG}$ at $180^{\circ} \mathrm{C}$ for 20 min led to $63.4 \%(\mathrm{w} / \mathrm{w})$ xylan removal from the pretreated solids. The hydrolyzates were pooled and concentrated and the resultant XOS concentrate was determined to have a total solid content of $71.2 \pm 0.3 \%(\mathrm{w} / \mathrm{w})$. In previous work, $\mathrm{MxG}$ autohydrolysis in a $0.6 \mathrm{~L}$ stainless Parr reactor, at temperatures ranging from $130^{\circ} \mathrm{C}$ to $150^{\circ} \mathrm{C}$ was reported to yield $40 \%$ (w/w) of initial xylan content (El Hage et al., 2010). In a stainless tube reactor heated at $160^{\circ} \mathrm{C}$ for $60 \mathrm{~min}, 65 \%(\mathrm{w} / \mathrm{w})$ of xylan was recovered as xylose and XOS (Ligero et al., 2011). Autohydrolysis conditions reported in the current work, provided comparable yields for the production of crude XOS concentrate. 
The carbohydrate composition of the XOS concentrate, adjusted for moisture content, was determined to be $39.8 \pm 2.1 \%$ xylose, $4.4 \pm 0.1 \%$ glucose, $5.1 \pm 0.1 \%$ arabinose and $2.7 \pm 0.3 \%$ galactose. The total xylose oligomer content, determined as per equation (1), was $30.1 \pm 1.0 \%$. Distribution of XOS (DP2 to DP6) in the concentrate varied from 0.6 to $15.9 \%$ dry weight, as shown in Table 1 and it was determined that DP2 to DP6 constituted $95.5 \%$ of the total XOS. The additional $4.5 \%$ may be made up of higher DPs of xylose or conjugates of xylan, arabinan, galactan and/or glucan. The total phenolic content of the concentrate was $4.1 \pm 0.2 \%$ gallic acid equivalent. Presence of phenolic compounds indicated that a certain degree of lignin degradation had occurred during the autohydrolysis reaction. It was calculated that in order to produce $1 \mathrm{~g}$ of the XOS concentrate, autohydrolysis of $3.7 \mathrm{~g}$ of MxG biomass was required. Conversely, $1 \mathrm{~g}$ of the concentrate used for CPC fractionation contained, $159.2 \mathrm{mg}$ of DP2, 59.0 $\mathrm{mg}$ of DP3, $56.0 \mathrm{mg}$ of DP4, 8.1 $\mathrm{mg}$ of DP5 and 5.6 mg of DP6.

\subsection{XOS separation and purification by CPC}

The XOS present in the concentrate were separated using CPC, generating 284 fractions. The ELSD chromatogram corresponding to a typical separation is presented in Figure 1; fractions corresponding to the highlighted area under the curve were consolidated. The HPLCRID chromatograms of the original concentrate and the CPC consolidated fractions are presented in Figure 2, the average yields of XOS, calculated on a dry weight basis of the concentrate, as well as the corresponding purities are presented in Table 2. Recovery of DP2 to DP6 oligomers varied between 61.9 to $90.2 \%$, while purity fluctuated between 31.5 to $63.2 \%$. The recovery of CPC fractionated XOS was the highest for DP2 at $90.2 \%$, based on the original composition of XOS concentrate (Table 2). The consolidated DP2 and DP3 fractions had comparatively higher purities of $61.9 \%$ and $63.2 \%$, respectively. The purities of CPC fractionated and consolidated 
MxG XOS were calculated based on their total mass fractions (Eqn. 2). In earlier reports, purities of XOS produced from purified xylan were higher, but were calculated based on area under the (chromatogram) curve, relative to other DPs and not on a mass basis (Qing et al., 2010; Lau et al., 2013)

\subsection{ESI-MS verification of CPC separated fractions}

Qualitative analysis of the CPC fractionated DP2 and DP3, performed using ESI-MS, was useful to verify their identity. As evidenced in Figure 3a, the major signals in consolidated DP2 fractions were $m / z 305.1[\mathrm{M}+\mathrm{Na}]^{+}$and $321.1[\mathrm{M}+\mathrm{K}]^{+}$which corresponded to individual pentobiose molecule bound with sodium and potassium ions. Similarly, the major signals in consolidated DP3 fractions were $\mathrm{m} / z 437.1[\mathrm{M}+\mathrm{Na}]^{+}$and $453.1[\mathrm{M}+\mathrm{K}]^{+}$(Figure 3b) which corresponded to individual pentotriose molecule bound with sodium and potassium ions. The ESI-MS spectrums validated that the DP2 and DP3 were isolated and were the main component in each fraction.

\section{Discussion}

Production of XOS for nutraceutical applications is a topic that is garnering research efforts. Low molecular weight XOS of sizes DP2 to DP5, at $70 \%$ purity, were reportedly fermented in vitro as well as in vivo, by prebiotic organisms belonging to the Bifidobacterium spp. (Hopkins et al., 1998). In one particular study, $90 \%$ of DP3 and $84 \%$ of DP2 were preferentially utilized by Bifidobacterium adolescentis (Gullón et al., 2008). Similarly certain strains of Lactobacillus spp. were reported to preferentially utilize xylose DP2 to DP4 when compared to other oligosaccharides, during mixed acid fermentations (Kontula et al., 1998). There are several enzymatic and chemical hydrolysis methods reported for the production of 
XOS from lignocellulosic biomass. However, few reports are addressing fractionation practices. Cara et al. (2012), described the production and separation of glucose and XOS by gel filtration chromatography, but did not separate XOS into individual DP components. Jayapal et al. (2013), extracted hemicellulose from sugarcane bagasse and produced XOS using xylanases. They detected their product with reducing sugar and thin layer chromatography assays, with no report on fractionation. Recently Mandelli et al. (2014), reported on the production of XOS from sugarcane bagasse, detecting and quantifying their products by capillary electrophoresis and HPAEC-PAD detection; however, this group also did not attempt to purify the xylan DPs.

Chen et al. (2014) originally used $10 \%$ activated carbon adsorption followed by aqueous ethanol elution and obtained a theoretical maximum recovery of $47.9 \%$ of XOS DP2 to DP9, from Miscanthus $x$ giganteus biomass. However, fractionation and purification of DPs into individual components was not attempted. Lau et al. (2011) were the first to report the use of CPC in separation and purification of XOS from acid hydrolyzed birchwood xylan: best results were obtained for the separation of xylose DP2 to DP5. With the adoption of a 4:1:4 volumetric ratio of butanol, methanol and water system, Lau et al. (2013) recovered $12.5 \mathrm{mg} / \mathrm{g}$ xylan of $81.2 \%$ pure xylobiose, $9.6 \mathrm{mg} / \mathrm{g}$ xylan of $71.0 \%$ pure xylotriose, $14.2 \mathrm{mg} / \mathrm{g}$ xylan of $62.4 \%$ pure xylotetraose and $21 \mathrm{mg} / \mathrm{g}$ xylan of $51.9 \%$ pure xylopentaose from autohydrolyzed birchwood xylan. Fractionating crude MxG hydrolyzate, containing other carbohydrates and lignin derivate, increased the complexity of separation and therefore reduced the purity of CPC fractions.

Lau et al. (2013), used CPC to recover $59.1 \mathrm{mg} / \mathrm{g}$ of DP2 to DP5, from autohydrolyzed birchwood xylan, and Bunnell (2013) reported CPC recoveries of $138 \mathrm{mg} / \mathrm{g}$ of DP2 to DP6 from alkali extracted switchgrass xylan. In this study, $230 \mathrm{mg}$ of xylose DP2 to DP6 were purified from $1 \mathrm{~g}$ of autohydrolyzed XOS concentrate, by CPC using a similar butanol- methanol-water 
solvent system. This work was on purifying XOS directly from a crude autohydrolyzed concentrate, illustrating that this technique can fractionate DPs from complex matrices. To our knowledge this is a first report on XOS purification from crude biomass hydrolyzates, using CPC.

\section{Conclusion}

XOS were purified from autohydrolyzed $\mathrm{MxG}$ concentrate by CPC with a solvent system composed of 4:1:4 (v/v/v) butanol, methanol and water. Through the collection of samples following elution order, $80 \%$ of DP2 to DP6 were fractionated from the original concentrate. Approximately, 90.2\% DP2, 64.5\% DP3, 71.2\% DP4, 61.9\% DP5 and 68.9\% of DP6 were recovered. Purities of each oligomer fraction was $61.9 \%$ DP2, 63.3\% DP3, 44.5\% DP4, 31.5\% DP5 and 51.3\% for DP6. The DP2 and DP3 fractions were validated by electrospray ionization mass spectrometry analysis. CPC thus provides a fast and effective means of separating XOS, from biorefinery by-products, for commercial applications.

\section{Acknowledgments}

In addition to the Arkansas State wide Mass Spectrometry facility (COBRE Grant Number: NIH P30 GM103450), University of Arkansas, Fayetteville, AR, the authors thank the Department of Energy award number GO88036, Arkansas Center for Plant Powered Production (P3), the Department of Biological and Agricultural Engineering and the Graduate School at the University of Arkansas for their funding and support. Authors are grateful to the Graduate School at the University of Arkansas for providing support to Ms. Kala Rajan through the Indian Women Fellowship during the course of these studies. The P3 Center is funded through the RII: 
Arkansas ASSET Initiatives (AR EPSCoR) I (EPS-0701890) and II (EPS-1003970) by the

National Science Foundation and the Arkansas Science and Technology Center.

\section{References}

Ainsworth, E.A., Gillespie, K.M. 2007. Estimation of total phenolic content and other oxidation substrates in plant tissues using Folin-Ciocalteu reagent. Nat. Protoc. 2, 875-877.

Attoumbre, J., Lesur, D., Giordanengo, P., Baltora-Rosset, S. 2012. Preparative separation of glycoalkaloids alpha-solanine and alpha-chaconine by centrifugal partition chromatography. J. Chromatogr. B. Analyt. Technol. Biomed. Life Sci. 908, 150-154.

Berthod, A., Armstrong, D.W. 1988. Centrifugal Partition Chromatography. I. General Features. J. Liq. Chromatogr. 11, 547-566.

Brosse, N., Dufour, A., Meng, X., Sun, Q., Ragauskas, A. 2014. Miscanthus: a fast-growing crop for biofuels and chemicals production. Biofuels Bioprod. Biorefining. 6, 580-598.

Brown, T.R., Brown, R.C. 2013. A review of cellulosic biofuel commercial-scale projects in the United States. Biofuels Bioprod. Biorefin. 7, 235-245.

Bunnell, K. 2013. Characterization and quantification of monomers, oligomers, and by-products from switchgrass hemicelluloses during biomass pretreatment. In: Biological and Agricultural Engineering, pp. 136, University of Arkansas, Fayetteville, AR.

Bunnell, K., Lau, C.S., Lay, J.O., Gidden, J. and Carrier, D.J. 2015. Production and fractionation of xylose oligomers from switchgrass hemicelluloses using centrifugal partition chromatography. J. Liq. Chromatogr. Relat. Technol. 38, 801-809. 
Cara, C., Ruiz, E., Carvalheiro, F., Moura, P., Ballesteros, I., Castroa, E., Gírio, F. 2012.

Production, purification and characterisation of oligosaccharides from olive tree pruning autohydrolysis. Ind. Crops Prod. 40, 225-231.

Carvalho, A.F.A., Neto, P.d.O., da Silva, D.F., Pastore, G.M. 2013. Xylo-oligosaccharides from lignocellulosic materials: Chemical structure, health benefits and production by chemical and enzymatic hydrolysis. Food Res. Int. 51, 75-85.

Chen, M.H., Bowman, M.J., Dien, B.S., Rausch, K.D., Tumbleson, M.E., Singh, V. 2014. Autohydrolysis of Miscanthus $x$ giganteus for the production of xylooligosaccharides (XOS): kinetics, characterization and recovery. Bioresour. Technol. 155, 359-365.

El Hage, R., Chrusciel, L., Desharnais, L., Brosse, N. 2010. Effect of autohydrolysis of Miscanthus x giganteus on lignin structure and organosolv delignification. Bioresour. Technol. 101, 9321-9329.

Gullón, P., González-Muñoz, M.J., van Gool, M.P., Schols, H.A., Hirsch, J., Ebringerová, A., Parajó, J.C. 2008. Assessment on the fermentability of xylooligosaccharides from rice husks by probiotic bacteria. J. Agric. Food. Chem. 56, 7482-7487.

Gullón, P., González-Muñoz, M.J., van Gool, M.P., Schols, H.A., Hirsch, J., Ebringerová, A., Parajó, J.C. 2010. Production, refining, structural characterization and fermentability of rice Husk Xylooligosaccharides. J. Agric. Food. Chem. 58, 3632-3641.

Hopkins, M.J., Cummings, J.H., Macfarlane, G.T. 1998. Inter-species differences in maximum specific growth rates and cell yields of bifidobacteria cultured on oligosaccharides and other simple carbohydrate sources. J. Appl. Microbiol. 85, 381-386. 
Jayapal, N., Samanta, A.K., Kolte, A.P., Senani, S., Sridhar, M., Suresh, K.P., Sampath, K.T. 2013. Value addition to sugarcane bagasse: Xylan extraction and its process optimization for xylooligosaccharides production. Ind. Crops Prod. 42, 14-24.

Jeon, J.S., Kim, S.-M., Lee, H.J., Um, B.H., Kim, H.K., Kim, C.Y. 2012. Preparative isolation and purification of prenylated isoflavanoids from Cudrania tricuspidata using centrifugal partition chromatography. J. Liq. Chromatogr. Relat. Technol. 35, 16071615.

Khullar, E., Dien, B.S., Rausch, K.D., Tumbleson, M.E., Singh, V. 2013. Effect of particle size on enzymatic hydrolysis of pretreated Miscanthus. Ind. Crops Prod. 44, 11-17.

Kim, T.H., Kim, T.H. 2014. Overview of technical barriers and implementation of cellulosic ethanol in the U.S. Energy. 66, 13-19.

Kontula, P., von Wright, A., Mattila-Sandholm, T. 1998. Oat bran beta-gluco- and xylooligosaccharides as fermentative substrates for lactic acid bacteria. Int. J. Food Microbiol. 45, 163-169.

Lau, C.S., Clausen, E.C., Lay, J.O., Gidden, J., Carrier, D.J. 2013. Separation of XOS using centrifugal partition chromatography with a butanol-methanol-water system. J. Ind. Microbiol. Biotechnol. 40, 51-62.

Lau, C.S., Bunnell, K.A., Clausen, E.C., Thoma, G.J., Lay, J.O., Gidden, J., Carrier, D.J. 2011. Separation and purification of XOS using centrifugal partition chromatography. J. Ind. Microbiol. Biotechnol.. 38, 363-370.

Ligero, P., van der Kolk, J.C., de Vega, A., van Dam, J.E.G. 2011. Production of xylooligosaccharides from Miscanthus x giganteus by autohydrolysis. BioResources. 6, 4417-4429. 
Mandelli, F., Brenelli, L.B., Almeida, R.F., Goldbeck, R., Wolf, L.D., Hoffmam, Z.B., Rullera, R., Rocha, G.J.M., Mercadante, A.Z., Squin, F.M. 2014. Simultaneous production of xylooligosaccharides and antioxidant compounds from sugarcane bagasse via enzymatic hydrolysis. Ind. Crops Prod. 52, 770-775.

Moure, A., Gullon, P., Domınguez, H., Parajo, J.C. 2006. Advances in the manufacture, purification and applications of xylo-oligosaccharides as food additives and nutraceuticals. Process Biochem. 41, 1913-1923.

Qing, Q., Yang, B., Wyman, C.E. 2010. Xylooligomers are strong inhibitors of cellulose hydrolysis by enzymes. Bioresour. Technol. 101, 9624-9630.

Sluiter, A., Hames, B., Hyman, D., Payne, C., Ruiz, R., Scarlata, C., Sluiter, J., Templeton, D., Wolfe, J. 2008a. Determination of total solids in biomass and total dissolved solids in liquid process samples National Renewable Energy Laboratory, Golden, CO.

Sluiter, A., Hames, B., Ruiz, R., Scarlata, C., Sluiter, J., Templeton, D. 2008b. Determination of sugars, byproducts, and degradation products in liquid fraction process samples National Renewable Energy Laboratory, Golden, CO.

Sluiter, A., Hames, B., Ruiz, R., Scarlata, C., Sluiter, J., Templeton, D., Crocker, D. 2008c. Determination of structural carbohydrates and lignin in biomass. In: NREL Laboratory Analytical Procedure, National Renewable Energy Laboratory, Golden, Colorado.

Somerville, C., Youngs, H., Taylor, C., Davis, S.C., Long, S.P. 2010. Feedstocks for lignocellulosic biofuels. Science. 329, 790-792.

Vázquez, M.J., Alonso, J.L., Domínguez, H., Parajó, J.C. 2000. Xylooligosaccharides: manufacture and applications. Trends Food Sci. Technol. 11, 387-393. 


\section{Captions to Tables:}

Table 1. Composition of autohydrolyzed XOS concentrate determined using HPLC analysis

Table 2. Yields of MxG XOS during centrifugal partition chromatography separation. 
Table 1. Composition of autohydrolyzed XOS concentrate determined using HPLC analysis

\begin{tabular}{cc}
\hline Compound & $\%$ Dry wt. ${ }^{\mathrm{a}}$ \\
\hline Xylobiose (DP2) & $15.9 \pm 1.5$ \\
Xylotriose (DP3) & $5.9 \pm 0.8$ \\
Xylotetraose (DP4) & $5.6 \pm 0.7$ \\
Xylopentaose (DP5) & $0.8 \pm 0.2$ \\
Xylohexaose (DP6) & $0.6 \pm 0.2$ \\
\hline \multicolumn{1}{c}{ Total } & $28.8 \pm 0.5$ \\
\hline${ }^{\mathrm{a}}$ Mean and standard deviation, $n=3$ &
\end{tabular}


Table 2. Yields of MxG XOS during centrifugal partition chromatography separation.

\begin{tabular}{lccc}
\hline Fractionated compounds & $\begin{array}{c}\text { Yield of each } \\
\text { compound } \\
(\mathrm{mg} / \mathrm{g})\end{array}$ & Recovery $^{\mathrm{b}}$ & Purity $^{\mathrm{c}}$ \\
\hline DP2-Xylobiose & $143.5 \pm 6.0$ & $90.2 \%$ & $61.9 \pm 1.9$ \\
DP3-Xylotriose & $38.1 \pm 5.0$ & $64.5 \%$ & $63.2 \pm 2.3$ \\
DP4-Xylotetraose & $39.8 \pm 1.0$ & $71.2 \%$ & $44.5 \pm 1.9$ \\
DP5-Xylopentaose & $4.9 \pm 0.1$ & $61.9 \%$ & $31.5 \pm 1.0$ \\
DP6-Xylohexaose & $3.9 \pm 0.3$ & $68.9 \%$ & $51.3 \pm 1.0$ \\
\hline a Mean and standard deviation $n=2$
\end{tabular}

${ }^{\mathrm{a}}$ Mean and standard deviation, $n=2$
$\mathrm{~b} \%$ Recovery was calculated based the theoretical maximum yield
${ }^{\mathrm{c}} \%$ Purity was calculated based on the total weight of each fraction 


\section{Figure captions}

Fig. 1. ELSD chromatogram of centrifugal partition chromatography separated XOS from autohydrolyzed $\mathrm{MxG}$ concentrate.

Fig. 2. HPLC-RID chromatogram of (a) original XOS concentrate and the consolidated XOS, (b) DP2 fraction, (c) DP3 fraction, (d) DP4 fraction, (e) DP5 fraction and (f) DP6 fraction

Fig. 3. ESI mass spectrum of consolidated xylose DP2 and DP3 purified from crude XOS concentrate by CPC. (a) DP2 fraction, (b) DP3 fraction 
Fig. 1

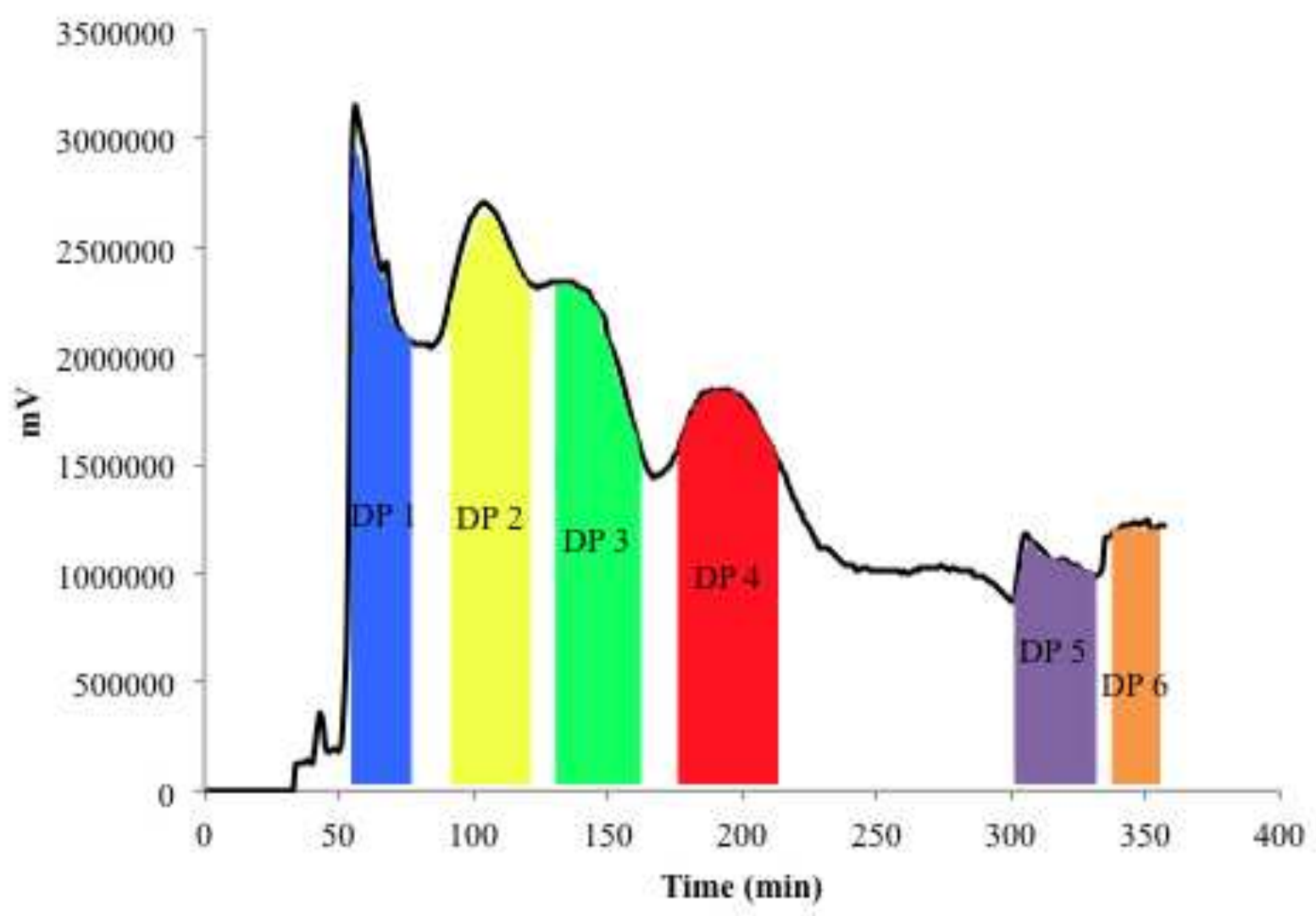

37

38

39

40

41

42

43

44

45

46

47

48

49

50

51

52

53

54

55

56

57

58

59

60

61

62

63

64

65 
Fig. 2

Auto-Scaled Zeroed Baseline Chromatogram
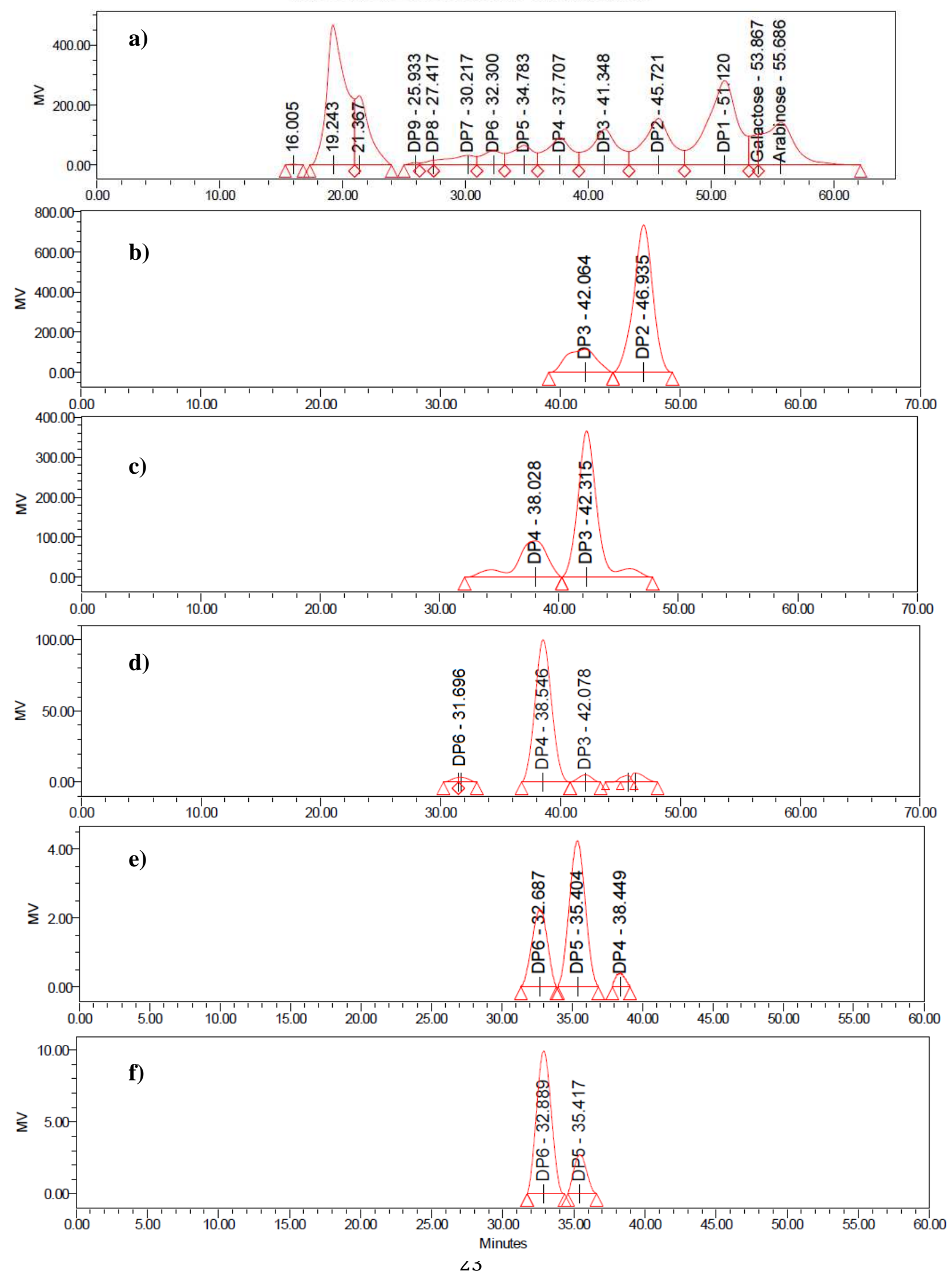
Fig. 3a.

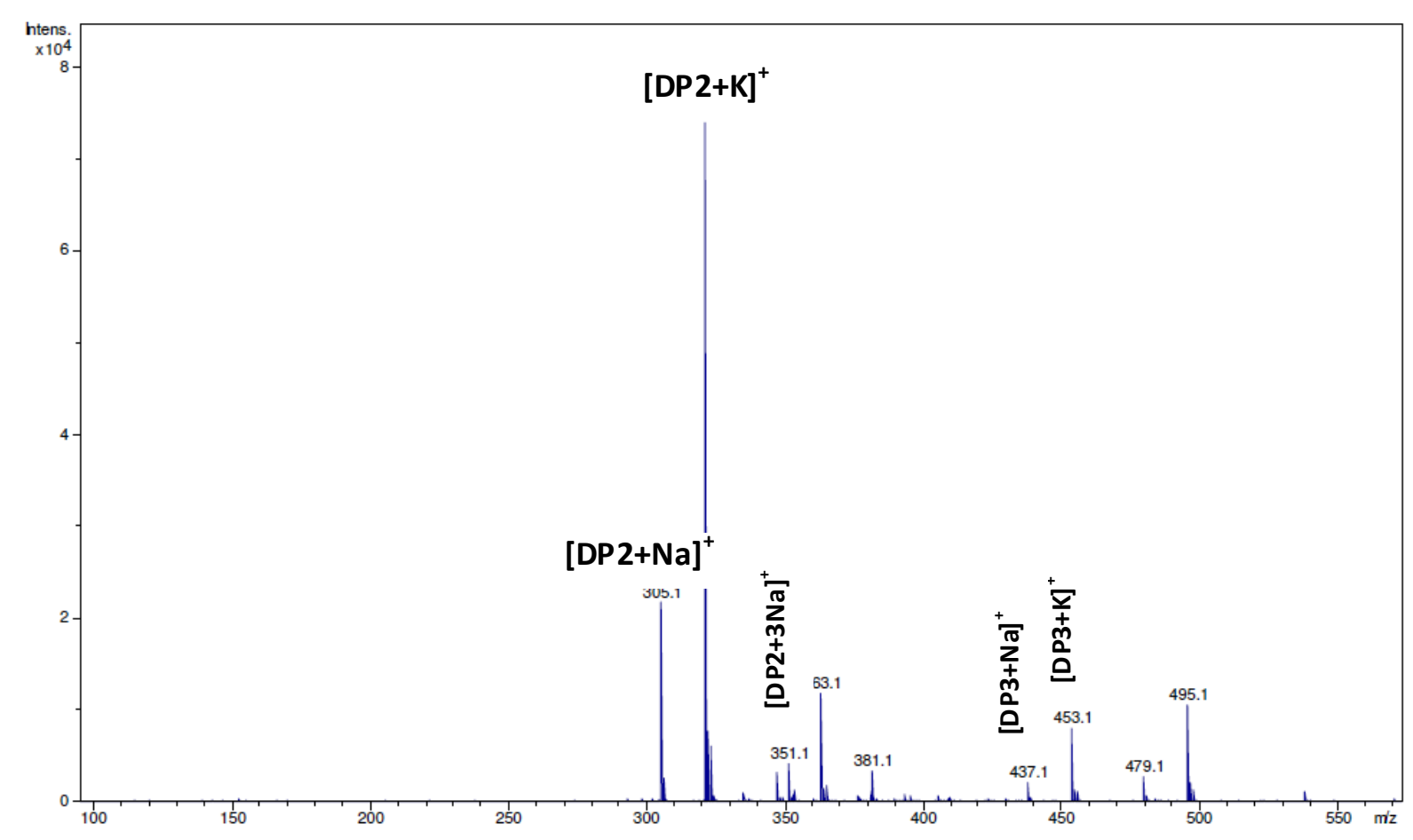

Fig. 3b.

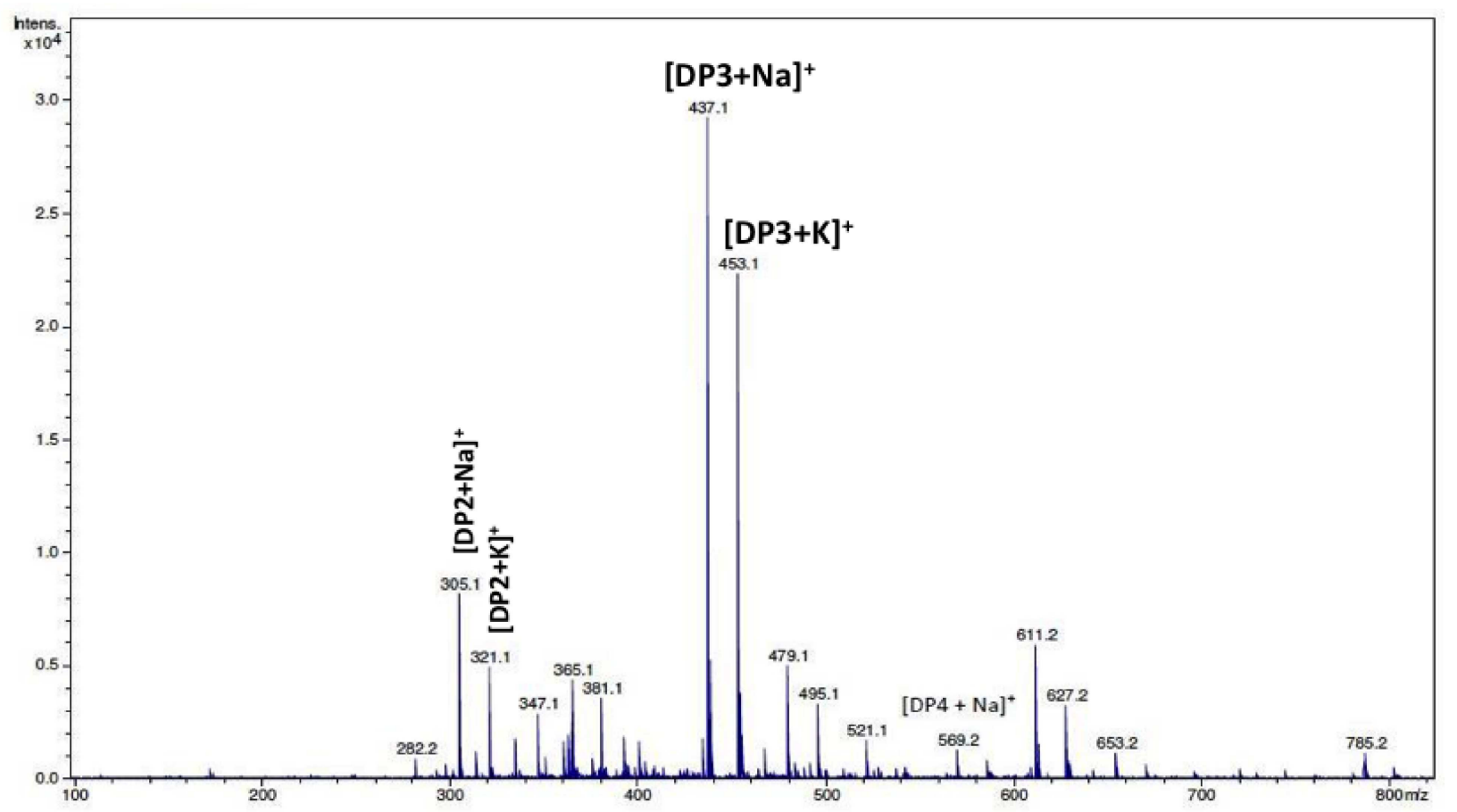

